\title{
Antipyretic and antinociceptive potential of extract/fractions of Potentilla evestita and its isolated compound, acacetin
}

\author{
Abdur Rauf ${ }^{1,2}$, Rehan Khan ${ }^{3}$, Haroon Khan ${ }^{4 *}$, Barkat Ullah ${ }^{5}$ and Samreen Pervez ${ }^{6}$
}

\begin{abstract}
Background: Fever and pain management is a very challenging job for the clinician as the available synthetic agents are causing serious side effects. The present research article deals with the antipyretic and antinociceptive activity of extract/fractions of Potentilla evestita and acacetin isolated from the chloroform fraction of the plant.

Methods: Various chromatographic and spectroscopic techniques were used for the isolation and characterizion of compound. In-vivo yeast induced fibrile mice were used for antipyretic activity while acetic acid induced writhing and formalin tests were used for antinociceptive.

Results: The extract/fractions of $P$. evestita caused marked antipyretic effect during various assessment times in which chloroform was the most prominent followed by ethyl acetate. When acacetin was injected, it produced marked effect with maximum activity of $33.28 \%$ and $55.01 \%$ at 5 and $10 \mathrm{mg} / \mathrm{kg}$ i.p respectively. When studied in acetic acid induced writhing test, the extract/fractions evoked significant antinociceptive effect in which chloroform was the most effective fraction followed by ethyl acetate. Acacetin showed significant antinociceptive effect with $44.77 \%$ and $67.03 \%$ reduction in abdominal constriction at 5 and $10 \mathrm{mg} / \mathrm{kg}$ i.p., respectively. Similarly, it evoked significant dose dependent reduction in noxious stimulation with $42.07 \%$ and $64.57 \%$ pain attenuation at 5 and $10 \mathrm{mg} / \mathrm{kg}$ i.p., respectively in initial phase. In the late phase, it illustrated more dominant effect with $46.32 \%$ and $67.29 \%$ reduction of painful sensation.
\end{abstract}

Conclusions: In conclusion, the extract/fractions of $P$. evestita as well as the isolated compound, acacetin showed strong antipyretic and antinociceptive activity in various animal models possibly mediated through both peripheral and central mechanism.

Keywords: Potentilla evestita, Acacetin, Antipyretic, Peripheral and central antinociceptive activity

\section{Background}

Potentilla evestita belong to family Rosaceae. It is distributed in the eastern Himalayan range from Indus to Kumaon, in Arctic, Alpine and temperate regions of the Northern hemisphere. P. evestita is a small perennial flowering herb. It has long hairy leaves with divided rootstock $[1,2]$. In Pakistan, it is usually found in Gilgit regions. In literature, several medicinal uses of $P$. evestita has been reported such as analgesic, antimicrobial, anti-inflammatory,

\footnotetext{
* Correspondence: hkdr2006@gmail.com

${ }^{4}$ Department of Pharmacy, Abdul Wali Khan University, Mardan 23200, Pakistan

Full list of author information is available at the end of the article
}

anti-diarrheal, anti-diabetic, hepatoprotective, anticancer, antispasmodic and ulcerative colitis [1].

Phytochemically, approximately 43 compounds have been isolated from $P$. evestita. The rhizomes of $P$. evestita contains rich amount of tannins i.e. 3.5\% hydrolysable tannins and $15-20 \%$ condensed tannins, pregnane derivative, 2,6 beta,7beta-trihydroxy-4-methyl-19-norpregna-1,3,5(10)trien-17-one, and pregnane derivative, 11alpha,17alpha,21trihydroxypregna-4,16(22)-diene-3,20-dione, have also isolated and reported from $P$. evestita $[3,4]$. The in-vivo antinociceptive and anti-inflammatory activities of umbelliferone isolated from the chloroform fraction of $P$. evestita is already reported [5]. The antinociceptive/antiinflammtory activity of acacetin, a bioflavonoid is already 
present in literature. Most probably it act by interfering with $5-\mathrm{HT}_{1 \mathrm{~A}}, \mathrm{GABA} / \mathrm{BDZs}$ and opioid receptors but not the NO-cGMP-K ${ }^{+}$channel pathway [6]. However, the antipyretic activity of Acacetin has not yet been explored experimentally.

To the best of our knowledge based on the available literature, no such study carried out on the antipyretic and antinociceptive activity of the extract/fractions of $P$. evestita. Keeping in mind the potential ethnopharmacological uses of the plant in said conditions, the effect of extract/fractions of $P$. evestita was studied in yeast induced febrile test and antinociceptive activity. The bioactivity guided isolation led to the isolation of acacetin from the chloroform fraction of $P$. evestita. The acacetin was also subjected to yeast induced fever test and antinociceptive activity in both peripherally acting and centrally acting antinociceptive paradigms.

\section{Methods}

\section{Plant specimen}

The whole plant of $P$. evestita $(15 \mathrm{~kg}$ ) was collected from Gilgit, Pakistan. The plant was identified by Taxonomist Department of Botany, University of Karachi. Voucher specimen (voucher No. 707) has been deposited in the herbarium of the Department of Botany, University of Karachi, Karachi, Pakistan (Herbarium No. 71212).

\section{Extraction and Isolation}

Shade dried whole plant (15 kg) of P. evestita was ground into fine powder and soaked in $25 \mathrm{~L}$ ethanol for 10 days at room temperature. The resulting extract was filtered and the filtrate evaporated under reduced pressure at $45^{\circ} \mathrm{C}$ to yield $300 \mathrm{~g}$ dark brown residue. The residue was suspended in water and subsequently extracted with solvents of increasing polarity, namely $n$-hexane $(3 \times 10 \mathrm{~L})$, chloroform $(3 \times 14 \mathrm{~L})$, ethyl acetate $(3 \times 12 \mathrm{~L})$ and methanol $(1 \times 3 \mathrm{~L})$. Each extract, evaporated under reduced pressure to afford $n$-hexane fraction (70 g), chloroform (75 g) EtOAc extract (8 g), and methanol (40 g). The chloroform fraction (60 g) was subjected to Column chromatography on silica gel (Merck Silica gel $60(0.063-0.200 \mathrm{~mm}), 5 \times 60 \mathrm{~cm})$. The column was first eluted with hexane-ethyl acetate $(100: 0 \rightarrow$ $0: 100)$ as the solvent system. A total of 100 fractions, RF-1 to RF-100 were obtained based on TLC profiles. Elution of the chromatogram with hexane- ethyl acetate $(100: 0 \rightarrow$ 100:0) gave the isolation of a known compound, acacetin (80 mg). The structure (Figure 1) of compound was confirmed by comparing the NMR and physical data with the reported data in literature [7].

\section{Animals}

BALB/c male mice (20-28 g) and Wistar rats (189222 g) were used. Animals were kept under standard laboratory condition at $25 \pm 2^{\circ} \mathrm{C}$. They were fed laboratory

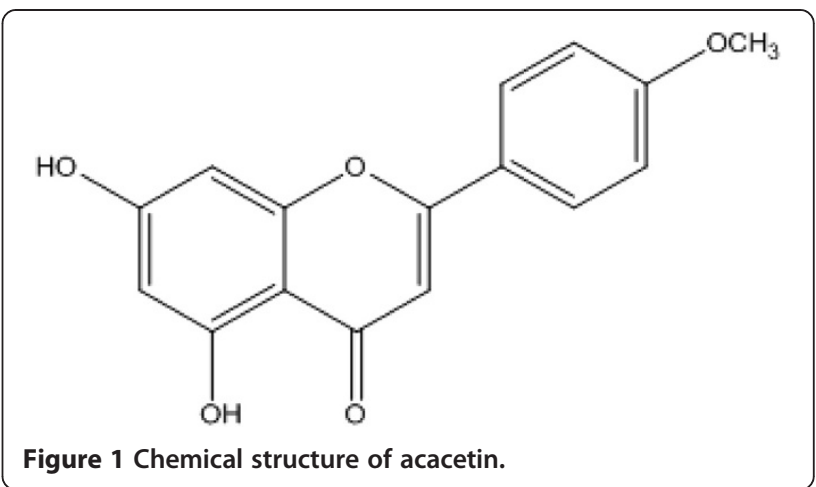

diet ad libitum and allowed free access to drinking water under standard environmental conditions of temperature $\left(25^{\circ} \mathrm{C}\right)$ in $12 \mathrm{~h}$ dark/12 h light control. The study was approved by the ethical committee of University of Karachi. Addtionally, all the experimental animals were treated according to ethical principles established by the University.

\section{Yeast induced fever test}

The antipyretic activity was determined in albino mice. The animals were divided in groups $(n=6)$. All groups were fasted overnight and allowed free accesses drinking water. Group one received saline as control group, group second received paracetamol as standard drug while the remaining groups received extract/fractions of $P$. evestita (50 and $100 \mathrm{mg} / \mathrm{i.p}$.) or acertain (5 and $10 \mathrm{mg} / \mathrm{kg}$ i.p.) The normal temperature was recorded using digital thermometer and then Fever was induced in all minces by injecting $20 \%$ aqueous suspension of Brewer's yeast $(10 \mathrm{ml} / \mathrm{kg}$ sc.). After $24 \mathrm{~h}$ rectal temperature was recorded and corresponding groups was injected with above doses. Rectal temperature was recorded periodically at $1,2,3,4$ and $5 \mathrm{~h}$ of drugs administration.

\section{Acetic acid induced writhing test}

Albino mice $(n=6)$ weighing $20-28$ g were used. All animals were withdrawn from food $2 \mathrm{~h}$ before the start of experiment and were divided in various groups. Group I was injected with normal saline $(10 \mathrm{ml} / \mathrm{kg})$ as control, Group II received standard drug diclofenac sodium (5 and $10 \mathrm{mg} / \mathrm{kg}$ ) while the remaining groups were injected extract/fractions of $P$. evestita (50 and $100 \mathrm{mg} / \mathrm{i} . \mathrm{p}$.) or acertain (5 and $10 \mathrm{mg} / \mathrm{kg}$ i.p.). After $30 \mathrm{~min}$ of the above treatment animals were treated i.p. with $1 \%$ acetic acid. The number of abdominal constrictions (writhes) was counted after $5 \mathrm{~min}$ of acetic acid injection for the period of $10 \mathrm{~min}[8,9]$.

\section{Formalin test}

In the formalin test, male wistar rats (189-222 g) were used following our previously reported method [10]. Briefly, the prescreened animals were arranged into groups 
$(n=6)$ which received either saline $(10 \mathrm{ml} / \mathrm{kg})$, compound (5 and $10 \mathrm{mg} / \mathrm{kg}$ i.p.). For the induction of pain, $0.05 \mathrm{ml}$ of formalin (2.5\% formaldehyde) was injected into the plantar surface of the right hind paw, 30 min after the treatment of all the animal groups, as described above. The nociceptive response was considered as the time spent by rat walking or can stand on injected paw; partially elevated paw; total elevation of injected paw, injected paw licking or biting. The first 0-5 min was computed as the first phase (neurogenic) and 25-30 min as last phase (anti-inflammatory) in the assay. Tramadol ${ }^{\mathrm{R}}$ (30 mg/kg i.p.) was used as a standard drug.

\section{Statistical analysis}

Values are shown as mean values \pm SEM of at least six animals. One-way analysis of variance was used for comparison test of significant differences among groups followed by Dunnet's multiple-comparison post test. $P<0.05$ was considered as significant from control.

\section{Results}

Effect of extract/fractions and acacetin in yeast induced fever test

The percent antipyretic effect of extract/fractions of $P$. evestita is shown in Figure 2. The crude extract demonstrated significant attenuation of induced fever in dose dependent manner during various assessment times with maximum effect $3^{\text {rd }}$ of treatment. Upon fractionation, marked changes in effect were noted. Of the test articles, chloroform fraction was the most dominant fraction followed by ethyl acetate fraction; illustrated maximum effect after $3^{\text {rd }} \mathrm{h}$ of treatment. The percent antipyretic effect of compound at test doses in yeast induced fever test is presented in Figure 3. Post-treatment of compound showed significant effect after $3^{\text {rd }} \mathrm{h}$ of treatment that remained significant up to $5^{\text {th }} \mathrm{h}$ (Figure 3a). At $10 \mathrm{mg} / \mathrm{kg}$ i.p., the antipyretic effect was significant even after $1^{\text {st }} \mathrm{h}$ of drug administration that remained significant up to $5^{\text {th }}$ h (Figure $3 \mathrm{~b}$ ). However, the standard drug,
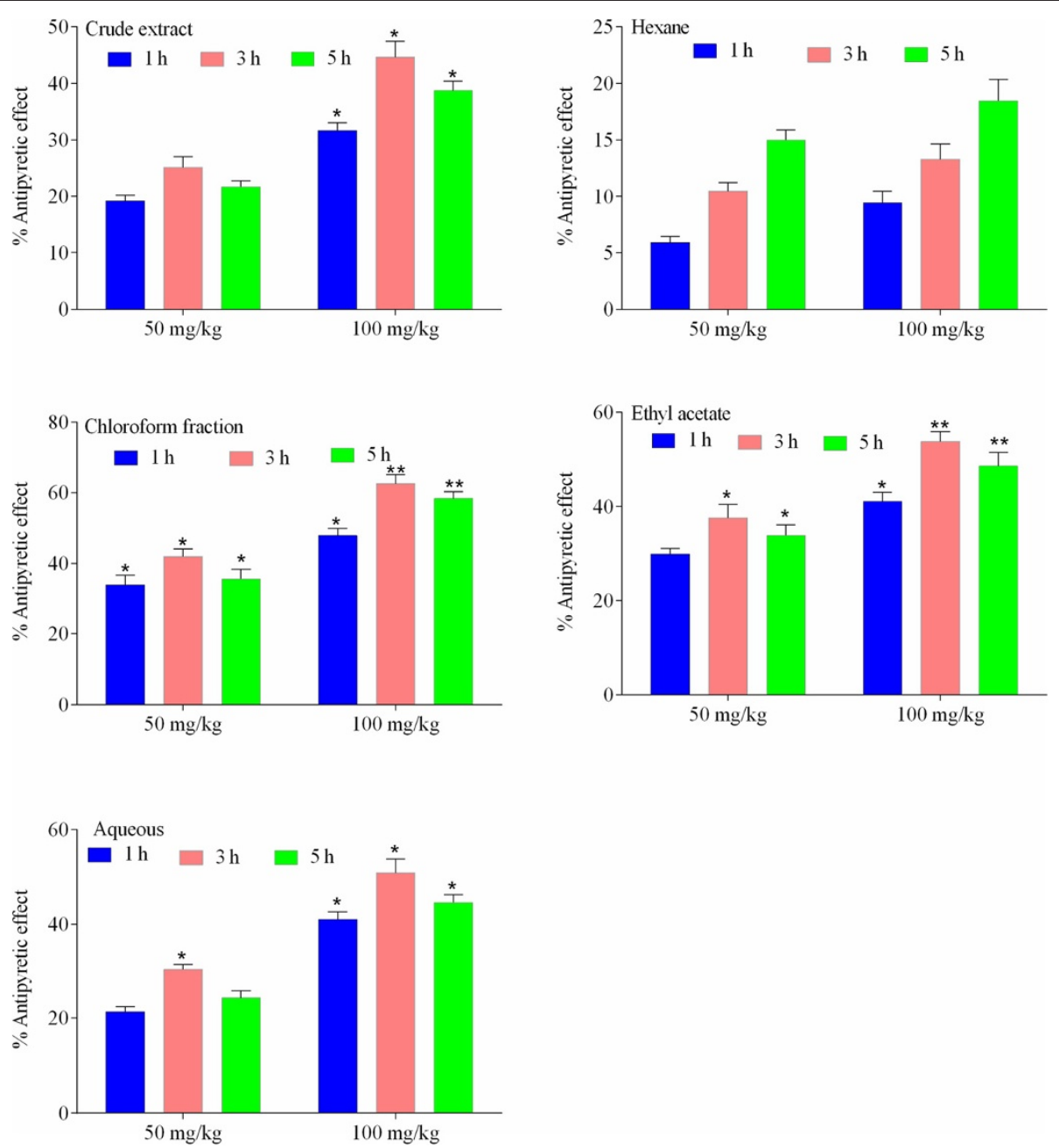

Figure 2 Antipyretic activity (\%) of crude extract/fractions in yeast induced fever test in mice. The data were analyzed by analysis of variance followed by Dunnett's test. Asterisks indicated statistically significant values from the control. ${ }^{*} p<0.05,{ }^{* *} p<0.01$. 


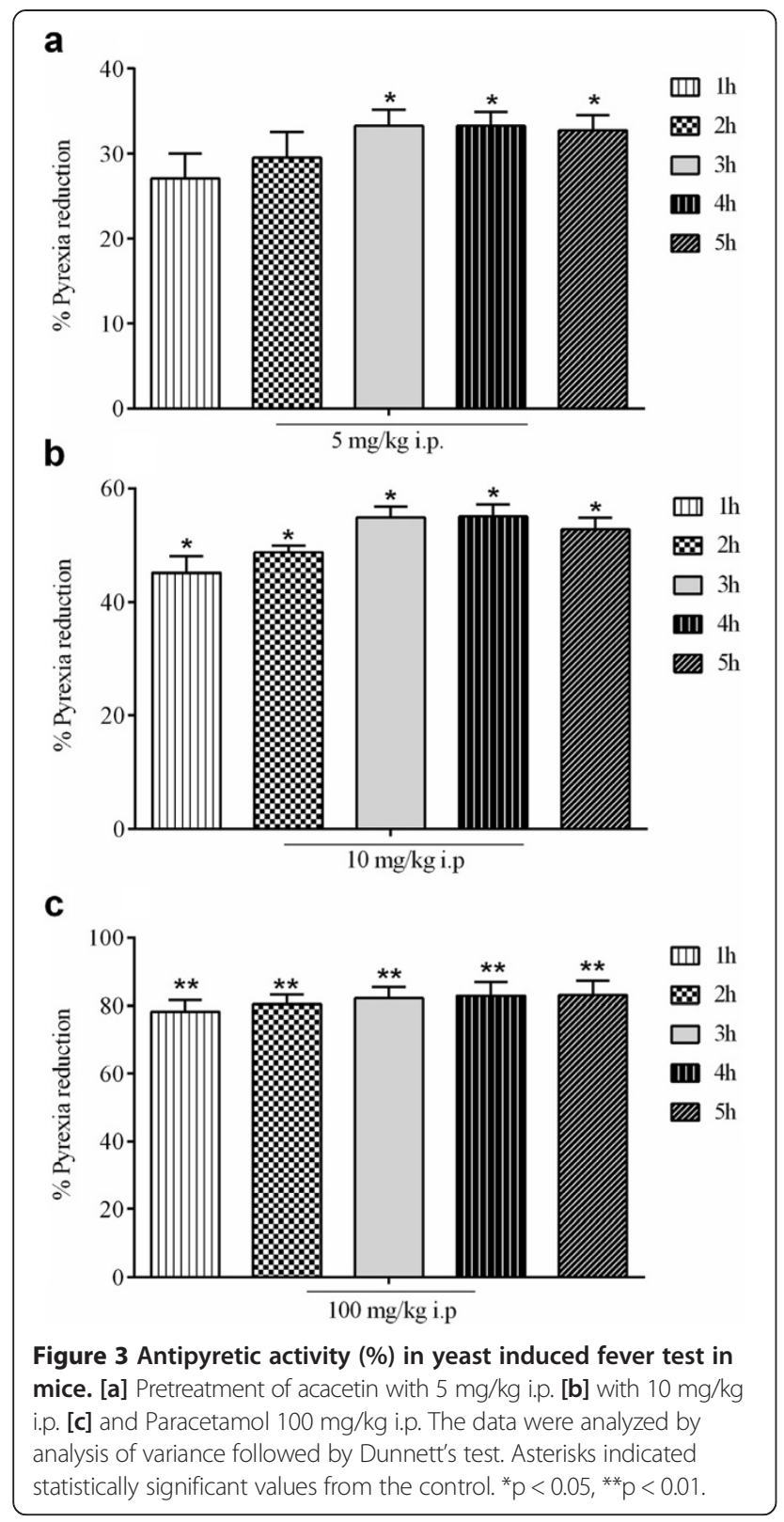

paracetamol showed most prominent effect during various assessment times (Figure 3c).

\section{Effect of extract/fractions and acacetin in acetic acid induced writhing test}

The antinociceptive effect of extract/fractions of $P$. evestita in acetic acid writhing test is presented in Figure 4. The crude extract provoked significant effect in dose dependent manner. However, fractionation produced marked changes in effect in which chloroform was the most prominent fraction followed by ethyl acetate. When acacetin was studied, it reduced pain perception in a concentration dependent manner. The reduction in abdominal

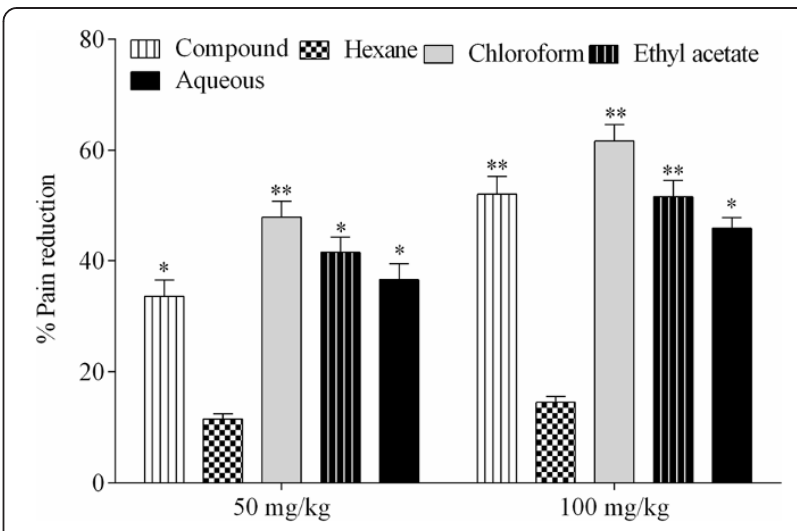

Figure 4 The effect (\%) of crude extract/fractions in acetic acid induced writhing test $(\boldsymbol{n}=6)$. The data were analyzed by analysis of variance followed by Dunnett's test. Asterisks indicated statistically significant values from the control. ${ }^{*} p<0.05,{ }^{* *} p<0.01$.

constriction was $44.77 \%$ and $67.03 \%$ at 5 and $10 \mathrm{mg} / \mathrm{kg}$ i.p., respectively. However, the standard compound, diclofenac exhibited more dominant effect with $55.56 \%$ and $78.45 \%$ at 5 and $10 \mathrm{mg} / \mathrm{kg}$ i.p., respectively (Figure 5).

\section{Effect of acacetin in formalin test}

The formalin-induced flinching behaviour was significantly attenuated by the injection of compound in the first phase (Figure 6). It produced significant dose dependent reduction in noxious stimulation with $42.07 \%$ and $64.57 \%$ pain reduction at 5 and $10 \mathrm{mg} / \mathrm{kg}$ i.p., respectively. In the late phase, it demonstrated more dominant effect with $46.32 \%$ and $67.29 \%$ blockade of painful sensation.

\section{Discussion}

The present study deals with the effect of crude extract/ fractions of $P$. evestita and bioactive activity guided isolation led to the isolation of acacetin from the chloroform soluble fraction followed by test in antipyretic and antihyperalgesic effects.

It is well established that the subcutaneous injection of brewer's yeast induces fever by increasing the synthesis of prostaglandin. It is considered as a useful test for the screening of plants materials as well as synthetic drugs for their antipyretic effect. The yeast-induced fever is called pathogenic fever and its etiology could be the production of prostaglandins [11,12]. The inhibition of prostaglandin synthesis could be the possible mechanism of antipyretic action as that of paracetamol and the inhibition of prostaglandin can be achieved by blocking the cyclooxygenase enzyme activity [5]. There are several mediators for fever and the inhibition of these mediators is responsible for the antipyretic effect $[11,13,14]$. The extract/fractions of the plant caused significant amelioration of the fribile mice in a dose dependent manner. 


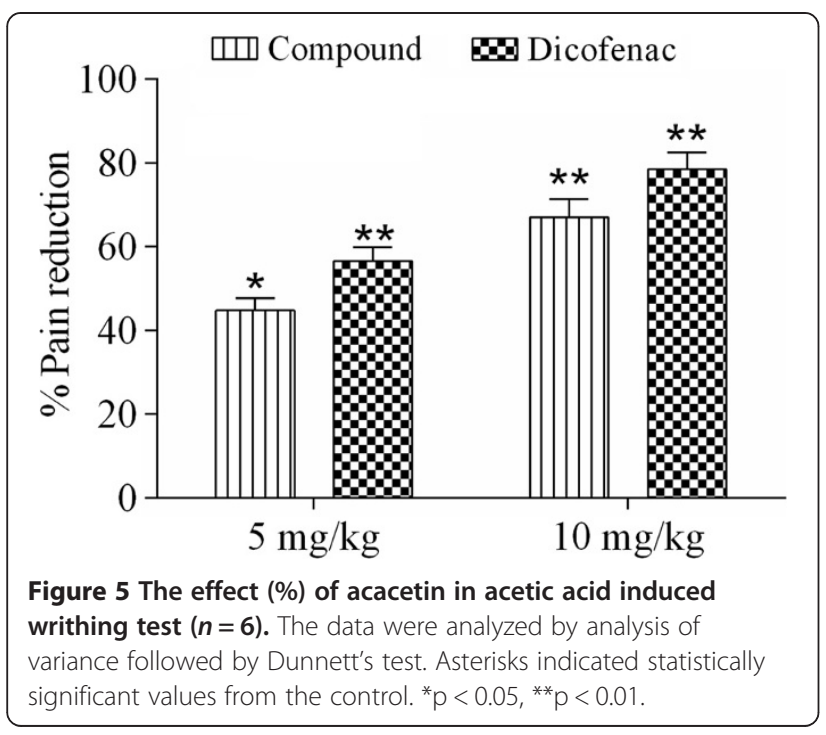

Among the fractions tested, chloroform was the most effective one followed by ethyl acetate. The intraperitoneal administration of isolated compound, strongly attenuated rectal temperature of yeast induced febrile mice. Thus it strongly complimented the antipyretic effect of extract/fractions of the plant and could be assumed that it interfered with the release or inhibit their activity of prostaglandins.

Of several in-vivo tests available for the evaluation of antinociceptive activity of natural compounds, two commonly used (acetic acid induced writhing and formalin induced flinching behavior) were employed [15]. The algesic mechanism for acetic acid induced writhing test has been characterized as liberation of different endogenous mediators $[13,16,17]$. The injection of acetic acid produced nociception in the form contraction of

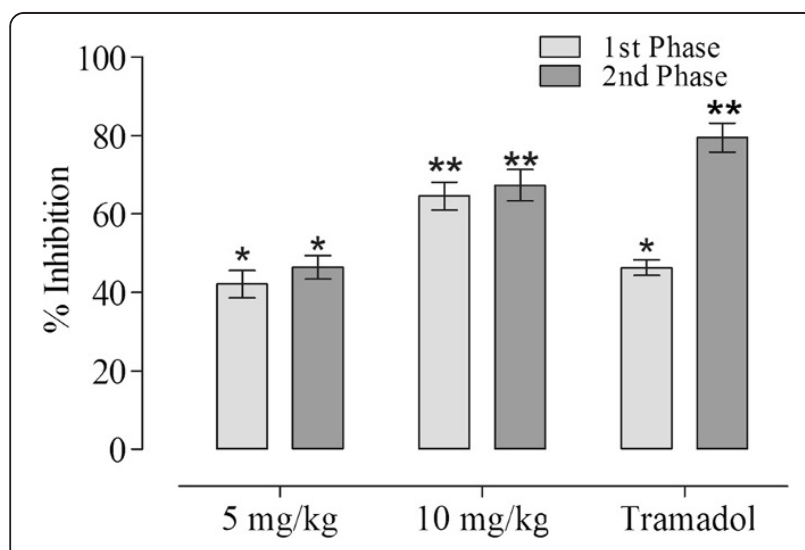

Figure 6 Protection (\%) of the acacetin in formalin induced flinching behaviour $(\boldsymbol{n}=\mathbf{6})$. The data were analyzed by analysis of variance followed by Dunnett's test. Asterisks indicated statistically significant values from the control. ${ }^{*} p<0.05,{ }^{* *} p<0.01$. the abdominal muscle accompanied by an extension of the forelimbs and body elongation. The extract/fractions of $P$. evestita exhibited profound antinociceptive effect in a dose dependent manner; with chloroform fraction as the most prominent fraction followed by ethyl acetate. The isolated compound significantly attenuated the abdominal constriction provoked by the acetic acid in a dose dependent manner. Thus one possible mechanism of antinociceptive activity of the extract/fractions of the plant as well as the isolated compound could be due to the blockade of the effect or the release of endogenous substances (arachidonic acid metabolites) that excite pain nerve endings.

In order to elucidate possible mechanism, the acetic acid induced writhing test is deficient in specificity; as different mechanism may be implicated in the reduction of muscular constriction for instance, sympathetic system through the release of biogenic amines, cyclooxygenases and their metabolites inhibition and through opioids receptors mechanisms [18]. This dearth was resolved while using the formalin test. This chemically induced noxious protocol is frequently used as a primary behavioral screening test for the assessment of the antinociceptive activity of compounds used in moderate, long lasting clinical pain [19]. Post formalin injection test produced a distinct biphasic response. The initial neurogenic phase (0-5 min) arises within seconds of formalin injection as a direct result of chemical stimulation of peripherally localized TRPA- 1 containing nociceptors $[10,15]$. The later tonic phase (25-30 min) occurs as a result of increased primary afferent drive with subsequent sensitization of nociceptive spinal neuron. It is observed that different analgesics may act differently $[11,20]$. The centrally acting drugs such as opioid inhibit both phases equally but peripherally acting drugs, such as cyclooxygenase inhibitors inhibit only the late phase [21,22].

The results of compound demonstrated strong antinociceptive in post-formalin induced flinching behaviour test. The effect was significant in both $1^{\text {st }}$ and $2^{\text {nd }}$ phases. Thus it is concluded that the peripheral effects were augmented by the central effects of the compound. However, details studies are most warranted to ascertain it further clinical utilization.

\section{Conclusions}

Finally, the extract/fractions of $P$. evestita showed marked antipyretic and antinociceptive effect in animal models which strongly augmented by the isolated compound, acacetin from the chloroform fraction. Thus our study provided scientific rationale to the folk uses of the plant as antipyretic and analgesic. Additionally, acacetin is a new candidate for further detail studies to ascertain its clinical use in further detail studies. 


\section{Competing interests}

The authors declare that they have no competing interest.

\section{Authors' contributions}

$A R$, RK and SP carried out experimental work. HK and BU written the paper. All authors read and approved the final manuscript.

\section{Acknowledgment}

We are extremely thankful to HEJ research institute of Karachi University for providing technical support.

\section{Author details}

${ }^{1}$ Institute of Chemical Sciences, University of Peshawar, Peshawar 25120, KPK, Pakistan. ${ }^{2}$ Geology Department, University of Peshawar, Peshawar, KPK, Pakistan. ${ }^{3} \mathrm{HEJ}$ Research Institute, Institute of Chemical and Biological sciences, Karachi University, Karachi, Pakistan. ${ }^{4}$ Department of Pharmacy, Abdul Wali Khan University, Mardan 23200, Pakistan. ${ }^{5}$ Department of Botany, University of Peshawar, Peshawar, KPK, Pakistan. ${ }^{6}$ Department of Pharmacy, University of Peshawar, Peshawar 25120, KPK, Pakistan.

Received: 13 June 2014 Accepted: 19 September 2014 Published: 18 November 2014

\section{References}

1. Tomczyk M, Latté KP: Potentilla-A review of its phytochemical and pharmacological profile. J Ethnopharmacol 2009, 122(2):184-204.

2. Anonymous: The Wealth of India. A Dictionary of Indian Raw Materials and Industrial Products Publication and Information Directorate. New Delhi: CSIR; 1998.

3. Rauf A, Khan R, Muhammad N: Antioxidant studies of various solvent fractions and chemical constituents of Potentilla evestita Th. Wolf. Afri J Pharm Pharmacol 2013, 7(39):2707-2710.

4. Khan R, Siddiq F, Fatima I, Yasmeen S, Karim A, Malik A, Afza N, Hameed S: Pregnane derivatives from Potentilla evestita. Nat Prod Comm 2011, 6(8):1083-1084.

5. Rauf A, Khanc R, Khand H, Perveze S, Pirzada AS: In vivo antinociceptive and anti-inflammatory activity of umbelliferone isolated from Potentilla evestita. Nat/ Prod Res 2014, 28(17):1371-1374.

6. Carballo-Villalobos A, González-Trujano M, Lopez-Muñoz F: Evidence of mechanism of action of anti-inflammatory/antinociceptive activities of acacetin. Euro J Pain 2014, 18:396-405.

7. Hayashi T, Asano S, Mizutani M, Takeguchi N, Kojima T, Okamura K, Morita $\mathrm{N}$ : Scopadulciol, an Inhibitor of Gastric $\mathrm{H}+, \mathrm{K}+-$ ATPhase from Scoparia dulcis, and Its Structure-Activity Relation-ships. J Nat Prod 1991, 54(3):802-809.

8. Muhammad N, Saeed M, Gilani SN, Ikram-ul-Haq, Khan H: Analgesic and anti-inflammatory profile of $\mathrm{n}$-hexane fraction of viola betonicifolia. Trop J Pharm Res 2013, 11(6):963-969.

9. Khan H, Saeed M, Khan MA, Khan I, Ashraf N: Antinociceptive activity of aerial parts of Polygonatum verticillatum: Attenuation of both peripheral and central pain mediators. Phytother Res 2011, 25(7):1024-1030.

10. Kaleem WA, Muhammad N, Qayum M, Khan H, Khan A, Aliberti L, De Feo V: Antinociceptive activity of cyclopeptide alkaloids isolated from Ziziphus oxyphylla Edgew (Rhamnaceae). Fitoterapia 2013, 91:154-158.

11. Muhammad N, Barkatullah, Ibrar M, Khan H, Saeed M, Khan AZ, Kaleem WA: In vivo screening of essential oils of Skimmia laureola leaves for antinociceptive and antipyretic activity. Asian Pac J Trop Biomed 2013, 3(3):202-206.

12. Wan J, Gong X, Jiang R, Zhang Z, Zhang L: Antipyretic and Anti-inflammatory Effects of Asiaticoside in Lipopolysaccharide-treated Rat through Up-regulation of Heme Oxygenase-1. Phytother Res 2013, 27(8):1136-1142.

13. Muhammad N, Saeed M, Khan H: Antipyretic, analgesic and antiinflammatory activity of Viola betonicifolia whole plant. BMC Compl Altern Med 2012, 12(1):59.

14. Khan H, Khan MA, Khan A, Tariq SA, Perviz S: Antipyretic activity of decoction of Joshanda and its saponin and sterol contents: Validation in an animal-based model. J Evidence-Based Compl Altern Med 2014, 19(2):99-103.

15. Khan H, Khan MA, Hussain S, Gaffar R, Ashraf N: In vivo antinociceptive and anticonvulsant activity of extracts of Heliotropium strigosum. Toxicol Indus Health 2013, doi:10.1177/0748233713513489.
16. Adebayo AH, John-Africa LB, Agbafor AG, Omotosho OE, Mosaku TO: Antinociceptive and anti-inflammatory activities of extract of Anchomanes difformis in rats. Pak J Pharm Sci 2014, 27(2):265-270.

17. Ahmed S, Sultana M, Hasan MMU, Azhar I: Analgesic and antiemetic activity of Cleome viscosa L. Pak J Bot 2011, 43(Special issue):119-122.

18. Hasnain F, Janbaz K, Qureshi M: Analgesic effect of ketamine and morphine after tonsillectomy in children. Pak J Pharm Sci 2012, 25(3):599-606

19. Olutoyin OT, Mati FG: Anti hyperalgesic potentials of Laggera aurita in Swiss Albino mice. Pak J Pharm Sci 2014, 27(1):169-172.

20. Rauf A, Muhammad N, Barkatullah KH, Abbas H: Antinociceptive, Sedative and Muscle Relaxants Activity of Caralluma tuberculata NE Brown. Orthop Muscular System 2013, 2(131):2161-0533.1000131.

21. Rauf A, Uddin G, Siddiqui BS, Muhammad N, Khan H: Antipyretic and antinociceptive activity of Diospyros lotus L. in animals. Asian Pac J Trop Biomed 2014, 4(Suppl 1):S382-S386.

22. Sałat K, Kulig K, Gajda J, Wieckowski K, Filipek B, Malawska B: Evaluation of anxiolytic-like, anticonvulsant, antidepressant-like and antinociceptive properties of new 2-substituted 4-hydroxy butanamides with affinity for GABA transporters in mice. Pharmacol Biochem Behav 2013, 110:145-153.

doi:10.1186/1472-6882-14-448

Cite this article as: Rauf et al:: Antipyretic and antinociceptive potential of extract/fractions of Potentilla evestita and its isolated compound, acacetin. BMC Complementary and Alternative Medicine 2014 14:448.

\section{Submit your next manuscript to BioMed Central and take full advantage of:}

- Convenient online submission

- Thorough peer review

- No space constraints or color figure charges

- Immediate publication on acceptance

- Inclusion in PubMed, CAS, Scopus and Google Scholar

- Research which is freely available for redistribution

Submit your manuscript at www.biomedcentral.com/submit
C Biomed Central 